\title{
Supersymmetry and Lorentz violation
}

\author{
M. S. Berger and V. Alan Kostelecký \\ Physics Department, Indiana University, Bloomington, Indiana 47405
}

(Received 28 December 2001; published 9 May 2002)

\begin{abstract}
Supersymmetric field theories can be constructed that violate Lorentz and $C P T$ symmetry. We illustrate this with some simple examples related to the original Wess-Zumino model.

DOI: 10.1103/PhysRevD.65.091701

PACS number(s): 11.30.Pb, 11.30.Cp, 11.30.Er
\end{abstract}

A major development in fundamental theoretical physics during the past century has been the understanding of the central role played by spacetime symmetries in nature. Conventional spacetime symmetries, including Lorentz and $C P T$ invariance, are now deeply ingrained in modern theories such as the standard model of particle physics and general relativity. Recent research includes investigations of larger spacetime symmetries, notably supersymmetry [1], and of the possibility that small violations of conventional spacetime symmetry could arise in an underlying theory at the Planck scale [2].

The essence of spacetime supersymmetry is the existence of transformations between bosons and fermions that yield a translation operator upon anticommutation:

$$
\left[P_{\mu}, P_{\nu}\right]=\left[P_{\mu}, Q\right]=0, \quad\{Q, \bar{Q}\}=2 \gamma^{\mu} P_{\mu},
$$

where the energy-momentum 4-vector $P_{\mu}$ generates spacetime translations, the spinor $Q$ generates supersymmetry transformations, and $\gamma^{\mu}$ are the Dirac matrices. Many supersymmetric Lorentz-invariant models exist. However, if supersymmetry is relevant to nature, experiment suggests it must be broken. Much of the phenomenology of supersymmetry conducted today is therefore within the context of the (minimal) supersymmetric standard model [3] in which soft supersymmetry-breaking but Lorentz-preserving interactions are added by hand. Soft interactions are superrenormalizable, while nonrenormalizable terms are taken to be suppressed by powers of the Planck scale or some other large scale associated with new physics. Soft terms can be motivated by studies of more fundamental theories and could arise from spontaneous breaking of supersymmetry [4]. Their physical implications at low energies can be analyzed in the framework of supersymmetric standard-model extensions including supersymmetry-breaking terms.

In a related vein, the physical implications of the breaking of conventional spacetime symmetries can be investigated using a general standard-model extension [5]. Its Lagrangian contains terms violating Lorentz and $C P T$ symmetry. Like the supersymmetry-breaking effects described above, these terms could arise from spontaneous symmetry violation. The nonlocal character of string theories offers a potential source for these terms with a natural origin in spontaneous Lorentz breaking [6] and provides strong motivation for investigating their physical implications at low energy. The renormalizable sector of the standard-model extension is a local field theory that would dominate Lorentz- and $C P T$-violating effects in low-energy physics. The requisite causality or positivity is- sues that result from treating the nonlocal underlying theory as a local field theory emerge at a high-energy scale determined by the Planck mass [7].

In this work, we consider an issue unaddressed in the literature: the existence of fully supersymmetric theories incorporating violation of Lorentz symmetry. For simplicity, we restrict attention here to global (rigid) supersymmetry and consider only renormalizable models conserving energy and momentum. A priori, even the existence of such theories is unclear, and in fact we find the simultaneous presence of supersymmetry and Lorentz violation provides a strong restriction on possible models.

For definiteness, we perform the analysis in the context of the Wess-Zumino model in four spacetime dimensions [8]. This model involves a scalar field $A$, a pseudoscalar $B$, a Majorana fermion $\psi$, an auxiliary scalar $F$, and an auxiliary pseudoscalar $G$. The associated Lagrangian $\mathcal{L}_{\text {WZ }}$ can be written as

$$
\mathcal{L}_{\mathrm{WZ}}=\mathcal{L}_{\mathrm{k}}+\mathcal{L}_{m}+\mathcal{L}_{g}
$$

where the kinetic, mass, and interaction terms are

$$
\begin{aligned}
& \mathcal{L}_{\mathrm{k}}=\frac{1}{2}\left(\partial_{\mu} A \partial^{\mu} A+\partial_{\mu} B \partial^{\mu} B+i \bar{\psi} \partial \psi+F^{2}+G^{2}\right), \\
& \mathcal{L}_{m}=m\left(-\frac{1}{2} \bar{\psi} \psi+A F+B G\right), \\
& \mathcal{L}_{g}=\frac{g}{\sqrt{2}}\left[F\left(A^{2}-B^{2}\right)+2 G A B-\bar{\psi}\left(A-i \gamma_{5} B\right) \psi\right] .
\end{aligned}
$$

To facilitate contact with existing studies of the Lorentzviolating standard-model extension, we adopt the conventions of Ref. [5] throughout this work.

Consider the special Lorentz-violating but CPTpreserving extension of the Wess-Zumino model given by the Lagrangian

$$
\mathcal{L}=\mathcal{L}_{\mathrm{WZ}}+\mathcal{L}_{\text {Lorentz }}
$$

where

$$
\begin{aligned}
\mathcal{L}_{\text {Lorentz }}= & k_{\mu \nu} \partial^{\mu} A \partial^{\nu} A+k_{\mu \nu} \partial^{\mu} B \partial^{\nu} B \\
& +\frac{1}{2} k_{\mu \nu} k_{\rho}^{\mu}\left(\partial^{\nu} A \partial^{\rho} A+\partial^{\nu} B \partial^{\rho} B\right) \\
& +\frac{1}{2} i k_{\mu \nu} \bar{\psi} \gamma^{\mu} \partial^{\nu} \psi .
\end{aligned}
$$

Without loss of generality, $k_{\mu \nu}$ can be taken as a real, symmetric, traceless, and dimensionless coefficient determining the magnitude of Lorentz violation, which is assumed small 
in the chosen observer inertial frame. The coefficient $k_{\mu \nu}$ transforms as a 2-tensor under observer Lorentz transformations changing the observer inertial frame but is unaffected by particle Lorentz transformations, which boost or rotate local field configurations within a fixed inertial frame [5].

Direct calculation reveals that the model given by Eqs. (4) and (5) is invariant up to a total derivative under the following set of modified infinitesimal supersymmetry transformations:

$$
\begin{aligned}
& \delta A=\bar{\epsilon} \psi, \quad \delta B=i \bar{\epsilon} \gamma_{5} \psi, \\
& \delta \psi=-\left(i \not b+i k_{\mu \nu} \gamma^{\mu} \partial^{\nu}\right)\left(A+i \gamma_{5} B\right) \epsilon+\left(F+i \gamma_{5} G\right) \epsilon, \\
& \delta F=-\bar{\epsilon}\left(i b+i k_{\mu \nu} \gamma^{\mu} \partial^{\nu}\right) \psi, \\
& \delta G=\bar{\epsilon}\left(\gamma_{5} b+k_{\mu \nu} \gamma_{5} \gamma^{\mu} \partial^{\nu}\right) \psi .
\end{aligned}
$$

In this equation, $\epsilon$ is a constant Majorana spinor. These transformation laws are observer covariant, so the supersymmetry is independent of the observer inertial frame. However, the presence of $k_{\mu \nu}$ implies the transformations are realized differently on particles with different orientations and boosts, as is to be expected in a theory with Lorentz violation. Note that the usual Wess-Zumino transformations are recovered in the limit $k_{\mu \nu} \rightarrow 0$.

The commutator of two supersymmetry transformations (6) yields

$$
\left[\delta_{1}, \delta_{2}\right]=2 i \bar{\epsilon}_{1} \gamma^{\mu} \epsilon_{2} \partial_{\mu}+2 i k_{\mu \nu} \bar{\epsilon}_{1} \gamma^{\mu} \epsilon_{2} \partial^{\nu},
$$

which involves the generator of translations. A modified supersymmetry algebra therefore exists. A superspace realization of this superalgebra is discussed below.

The Lagrangian (4) thus provides an explicit example of an interacting model with both exact supersymmetry and Lorentz violation. We know of no other supersymmetric, $C P T$-preserving, and Lorentz-violating extension of the minimal Wess-Zumino multiplet. The possible supersymmetry transformation laws are strongly restricted by various factors, including the linearity in $\epsilon$ and the fields, the small number of physical Lorentz-violating terms for Majorana spinors, the properties of coefficients for Lorentz violation, and the requirement of closure of the induced supersymmetry algebra.

The presence of $k_{\mu \nu}$ in the supersymmetric transformation forces a relationship on the coefficients for Lorentz violation in Eq. (5). This is analogous to the common mass and common couplings that are a standard consequence of supersymmetric theories. Without the supersymmetry, each of the five terms in Eq. (5) could have different coefficients, a variety that is reflected in the form of the general Lorentz-violating standard-model extension. Physical consequences of the relationship among the coefficients in Eq. (5) are to be expected. For example, the fermionic propagator is

$$
i S_{F}(p)=\frac{i}{p_{\mu}\left(\gamma^{\mu}+k_{\mu \nu} \gamma^{\nu}\right)-m} .
$$

Rationalizing the denominator of this propagator gives

$$
i S_{F}(p)=i \frac{p_{\mu}\left(\gamma^{\mu}+k_{\mu \nu} \gamma^{\nu}\right)+m}{p^{2}+2 p^{\mu} p^{\nu} k_{\mu \nu}+k_{\mu \rho} k^{\rho}{ }_{\nu} p^{\mu} p^{\nu}},
$$

using the symmetry of $k_{\mu \nu}$. Consequently, the scalar and fermionic propagators have the same structure. We therefore anticipate divergence cancellations and nonrenormalization theorems generalizing the usual results.

Note also that the Lorentz violation of the theory is physical. The interactions eliminate the possibility of a trivializing field redefinition [5]. If $\mathcal{L}_{g}$ were absent one might consider, for example, a simultaneous nonlocal field redefinition of the form $f(x) \rightarrow f\left(x^{\prime}\right)=\exp \left(\widetilde{k}_{\mu \nu} x^{\mu} \partial^{\nu}\right) f(x)$ of all fields in the supermultiplet, which for suitable $\widetilde{k}_{\mu \nu}$ would eliminate the terms in $\mathcal{L}_{\text {Lorentz }}$ while leaving unaffected the mass terms $\mathcal{L}_{m}$. However, with $\mathcal{L}_{g}$ present the same field redefinition merely replaces $\mathcal{L}_{\text {Lorentz }}$ with $x$-dependent Lorentz-violating interactions. Similarly, attempting to absorb the Lorentz violation into a redefinition of coordinates and momenta has no physical effect, merely moving the violation into the metric.

The Lorentz-violating $C P T$-preserving model (4) can be described in a superfield formulation. Define

$$
\phi=\frac{1}{\sqrt{2}}(A+i B), \quad \mathcal{F}=\frac{1}{\sqrt{2}}(F-i G) .
$$

In terms of these complex scalars, the left-chiral superfield appropriate for the model (4) is

$$
\begin{aligned}
\Phi(x, \theta)= & \phi(x)+\sqrt{2} \bar{\theta} \psi_{L}(x)+\frac{1}{2} \bar{\theta}\left(1-\gamma_{5}\right) \theta \mathcal{F}(x) \\
& +\frac{1}{2} i \bar{\theta} \gamma_{5} \gamma^{\mu} \theta\left(\partial_{\mu}+k_{\mu \nu} \partial^{\nu}\right) \phi(x) \\
& -\frac{i}{\sqrt{2}} \bar{\theta} \theta \bar{\theta}\left(b+k_{\mu \nu} \gamma^{\mu} \partial^{\nu}\right) \psi_{L}(x) \\
& -\frac{1}{8}(\bar{\theta} \theta)^{2}\left(\partial_{\mu}+k_{\mu \nu} \partial^{\nu}\right)^{2} \phi(x) .
\end{aligned}
$$

Here, the subscript $L$ denotes projection with $\frac{1}{2}\left(1-\gamma_{5}\right)$. The Lagrangian (4) can then be expressed as

$$
\mathcal{L}=\left.\Phi^{*} \Phi\right|_{D}+\left(\left.\frac{1}{2} m \Phi^{2}\right|_{F}+\left.\frac{1}{3} g \Phi^{3}\right|_{F}+\text { H.c. }\right),
$$

where the symbols $\left.\right|_{D}$ and $\left.\right|_{F}$ refer to projections onto the $D$ and $F$-type components of the (holomorphic) functions of $\Phi(x, \theta)$. The theory can therefore be represented as an action in superspace.

A superspace realization $Q$ of the supersymmetry generators can be obtained via a coset-space construction [9]. For a supersymmetry transformation on $\Phi(x, \theta)$ generated as $\delta_{Q} \Phi(x, \theta)=-i \bar{\epsilon} Q \Phi(x, \theta), Q$ is found to be

$$
Q=i \partial_{\bar{\theta}}-\gamma^{\mu} \theta \partial_{\mu}-k_{\mu \nu} \gamma^{\mu} \theta \partial^{\nu} .
$$

This induces the supersymmetry transformations (6) on the component fields in $\Phi(x, \theta)$.

The superalgebra generated by $Q$ and $P_{\mu}=i \partial_{\mu}$ is 


$$
\left[P_{\mu}, Q\right]=0, \quad\{Q, \bar{Q}\}=2 \gamma^{\mu} P_{\mu}+2 k_{\mu \nu} \gamma^{\mu} P^{\nu} .
$$

By virtue of the Lorentz violation, manifest through the presence of $k_{\mu \nu}$, this superalgebra lies outside the usual list of possible supersymmetric extensions of the Poincaré, de Sitter, or conformal algebras [10]. It appears feasible and would be of interest to obtain a general classification of such superalgebras allowing for the possibility of Lorentz violation.

As a more technical remark, we observe that a superfield covariant derivative $\mathcal{D}$ can be introduced in analogy with the usual case:

$$
\mathcal{D}=i \partial_{\bar{\theta}}+\gamma^{\mu} \theta \partial_{\mu}+k_{\mu \nu} \gamma^{\mu} \theta \partial^{\nu}
$$

It obeys

$$
\{\mathcal{D}, \overline{\mathcal{D}}\}=-2 \gamma^{\mu} P_{\mu}-2 k_{\mu \nu} \gamma^{\mu} P^{\nu},
$$

and has vanishing anticommutators with $Q, \bar{Q}$. The form of Eq. (16) implies the geometry of superspace is changed in that the torsion is modified by the presence of Lorentz violation. The right projection $\frac{1}{2}\left(1+\gamma_{5}\right) \mathcal{D}$ defines a left-chiral coordinate $x_{+}^{\mu}$ through the condition $\mathcal{D}_{R} x_{+}=0$ :

$$
x_{+}^{\mu}=x^{\mu}+\frac{1}{2} i \bar{\theta} \gamma_{5} \gamma^{\mu} \theta+\frac{1}{2} i k^{\mu \nu} \bar{\theta} \gamma_{5} \gamma_{\nu} \theta .
$$

In terms of $x_{+}^{\mu}$, the left-chiral superfield (11) takes the simpler form

$$
\Phi(x, \theta)=\phi\left(x_{+}\right)+\sqrt{2} \bar{\theta}_{R} \psi_{L}\left(x_{+}\right)+\bar{\theta}_{R} \theta_{L} \mathcal{F}\left(x_{+}\right),
$$

and is annihilated by $\mathcal{D}_{R}, \mathcal{D}_{R} \Phi\left(x_{+}, \theta\right)=0$.

The form (14) of the superalgebra involves the generator $P^{\mu}$ of translations. A conserved canonical energy-momentum tensor $\theta^{\mu \nu}$ can be constructed, and $P^{\mu}$ is then recovered as the spatial integral of the components $\theta^{0 \mu}[5,7]$. The presence of derivative couplings in $\mathcal{L}_{\text {Lorentz }}$ means that care is required in physical interpretation because the physically propagating supermultiplet differs by a field redefinition from the superfield components of $\Phi(x, \theta)$. Also, the 4-momenta for one-particle states obey modified dispersion laws. However,

$$
\left[Q, P^{2}\right]=0,
$$

so the eigenvalues of $P^{2}$ must be the same for members of the supermultiplet. Since the superpotential containing the mass and coupling terms is unaffected by the Lorentz violation, analogues should exist for various conventional results on supersymmetry breaking [11]. Note also that a supersymmetry current can be obtained because the supersymmetry is a continuous global symmetry of the Lagrangian. The existence of the superfield formulation implies a corresponding supercurrent superfield can be constructed.

In the context of spontaneous Lorentz violation in an underlying covariant string field theory, the coefficients $k_{\mu \nu}$ would be related to one or more vacuum expectation values of Lorentz vector or tensor fields [6]. The form of the transformations (6) then suggests that the supersymmetry must be realized in a nonlinear fashion in the underlying string field theory, since the coefficients $k_{\mu \nu}$ would be associated with dynamical fields. Note also that, even if a linear supersymmetry in the underlying string theory breaks along with Lorentz symmetry, the model (4) demonstrates that an exact linear supersymmetry could still exist in the effective lowenergy theory.

We next consider the more difficult challenge of constructing a $C P T$-violating extension of the Wess-Zumino model. It is a famous result of quantum field theory that a local Lorentz-invariant theory preserves the combination $C P T$ [12]. However, if Lorentz invariance is abandoned, one can consider the addition of a $C P T$-odd component to $\mathcal{L}_{\mathrm{WZ}}$,

$$
\mathcal{L}=\mathcal{L}_{\mathrm{WZ}}+\mathcal{L}_{\mathrm{CPT}},
$$

where

$$
\mathcal{L}_{\mathrm{CPT}}=k_{\mu}\left(A \partial^{\mu} B-B \partial^{\mu} A\right)+\frac{1}{2} k^{2}\left(A^{2}+B^{2}\right)-\frac{1}{2} k_{\mu} \bar{\psi} \gamma_{5} \gamma^{\mu} \psi .
$$

Here, the $C P T$ violation is controlled by $k_{\mu}$, which is a real coefficient of mass dimension one transforming as a vector under observer Lorentz transformations but as a collection of four scalars under particle Lorentz transformations. The terms (21) respect $C$ but violate $P$ or $T$, giving an overall $C P T$ violation. The terms with coefficient $k^{2}=k_{\mu} k^{\mu}$ represent mass renormalizations varying with the particle boost and orientation. This is necessary for the existence of the supersymmetry below, except in the special case of lightlike $k_{\mu}$.

The model (20) transforms into a total derivative under the infinitesimal supersymmetry transformations

$$
\begin{aligned}
& \delta A=\bar{\epsilon} \psi, \quad \delta B=i \bar{\epsilon} \gamma_{5} \psi, \\
& \delta \psi=-\left(i \not b+\gamma_{5} k\right)\left(A+i \gamma_{5} B\right) \epsilon+\left(F+i \gamma_{5} G\right) \epsilon, \\
& \delta F=-\bar{\epsilon}\left(i b-\gamma_{5} k\right) \psi, \\
& \delta G=\bar{\epsilon}\left(\gamma_{5} \not+i k\right) \psi .
\end{aligned}
$$

The uniqueness of this supersymmetry can be established on dimensional grounds. Note that it acts differently on the leftchiral multiplet and its conjugate, for example,

$$
\begin{aligned}
& \delta \psi_{L}=(-i \not b+k)(A+i B) \epsilon_{R}+(F-i G) \epsilon_{L}, \\
& \delta \psi_{R}=(-i \not b-k)(A-i B) \epsilon_{L}+(F+i G) \epsilon_{R} .
\end{aligned}
$$

The terms (21) emerge from $\mathcal{L}_{\mathrm{WZ}}$ via the redefinition

$$
\psi \rightarrow e^{-i \gamma_{5} k \cdot x} \psi, \quad(\phi, \mathcal{F}) \rightarrow e^{i k \cdot x}(\phi, \mathcal{F})
$$

The components of the left-chiral multiplet and its conjugate are therefore shifted by opposite position-dependent phases. The mass and coupling terms in $\mathcal{L}_{g}$ would acquire $C P T$ - and Lorentz-violating position-dependent coefficients under the field redefinition, so if energy-momentum is to be conserved they would need to be added afterwards. However, they are then inconsistent with the supersymmetry (22). The same is true of $P$-odd mass or coupling terms, such as the combina- 
tion $\left(i \bar{\psi} \gamma_{5} \psi+2 A G-2 B F\right)$. In the absence of noninvariant couplings, the field redefinition implies that the $C P T$ and Lorentz violation in Eq. (21) is unphysical.

Acting on the components of the left-chiral multiplet, the commutator of two supersymmetry transformations (22) gives

$$
\left.\left[\delta_{1}, \delta_{2}\right]\right|_{\text {left }}=2 i \bar{\epsilon}_{1} \gamma^{\mu} \epsilon_{2} \partial_{\mu}-2 k_{\mu} \bar{\epsilon}_{1} \gamma^{\mu} \epsilon_{2},
$$

which again involves the generator of translations. The last term is a special consequence of Lorentz violation, absent in the conventional spacetime superalgebras but allowed here because $k_{\mu}$ has mass dimension one. However, the commutator of two supersymmetry transformations on the rightchiral multiplet yields instead

$$
\left.\left[\delta_{1}, \delta_{2}\right]\right|_{\text {right }}=2 i \bar{\epsilon}_{1} \gamma^{\mu} \epsilon_{2} \partial_{\mu}+2 k_{\mu} \bar{\epsilon}_{1} \gamma^{\mu} \epsilon_{2} .
$$

The relative sign change in the last term complicates a superspace construction. It has features reminiscent of central charges for conventional extended supersymmetry. It would be interesting to obtain an explicit superspace formulation of this model with a differential realization of the supersymmetry transformations that reproduces the intertwined relations (25) and (26). In any case, however, there would be an obstacle to construction of an invariant superpotential involving the usual $F$-type terms: the $F$ term no longer transforms as a total derivative under a supersymmetry transformation, as follows from Eq. (22).

Although it lies beyond our present scope, it would be of interest to investigate the possibility of Lorentz-violating models with extended supersymmetry. Certainly, $N=1$ models similar to those in Eqs. (4) and (20) but involving several supermultiplets appear straightforward to construct. The presence of several multiplets might permit physical $C P T$ violation, although more general field redefinitions that mix fields between multiplets would need to be considered. It may also be useful to allow for variant multiplets in constructing Lorentz-violating models. For example, the scalar $\phi$ can be regarded as the dual of an antisymmetric 2-tensor, for which the extra spacetime indices might permit distinct Lorentz-violating couplings. Note also that various renormalizable Lorentz- and $C P T$-violating terms exist that are unused in the theories given above, including $\left(A^{2} \partial B\right.$ $\left.\pm B^{2} \partial A\right), \phi \bar{\psi} \gamma_{5} \gamma^{\mu} \psi$, and $\bar{\psi} \sigma^{\mu \nu} \partial^{\lambda} \psi$. We are unaware of any supersymmetric role for these terms, which have dimensionless coefficients for Lorentz and $C P T$ violation carrying one or three spacetime indices. In this sense, the theories given above appear unique.

One can extend the considerations discussed here to other representations of supersymmetry. For example, we expect the vector supermultiplet to have a Lorentz-violating generalization, so a supersymmetric Lorentz-violating extension of quantum electrodynamics should exist. Similarly, it appears feasible to construct a supersymmetric Lorentz-violating standard-model extension, in which case potentially realistic models could be obtained by including soft supersymmetrybreaking terms. These soft terms would include Lorentzviolating dimension-three operators of the types discussed in Ref. [5]. In the context of supergravity models, the scale $m$ of the soft terms is often related to the scale $M_{s}$ of supersymmetry breaking by $m \sim M_{s}^{(1+n)} / M_{P}^{n}$ for some integer $n>0$. Generalizing the results here to local supersymmetry and local Lorentz violation might therefore eventually uncover determining relationships among the scale of Lorentz violation, the scale of supersymmetry breaking, and the underlying Planck scale.

This work was supported in part by DOE grant DE-FG0291ER40661.
[1] For textbooks on the subject, see, for example, S. Weinberg, The Quantum Theory of Fields (Cambridge University Press, Cambridge, England, 2000), Vol. III; J. Wess and J. Bagger, Supersymmetry and Supergravity, 2nd ed. (Princeton University Press, Princeton, NJ, 1992).

[2] For reviews of various theoretical ideas, see, for example, $C P T$ and Lorentz Symmetry, edited by V.A. Kostelecký (World Scientific, Singapore, 1999).

[3] S. Dimopoulos and H. Georgi, Nucl. Phys. B193, 150 (1981); N. Sakai, Z. Phys. C 11, 153 (1981).

[4] For a review, see, for example, H.P. Nilles, Phys. Rep. 110, 1 (1984).

[5] D. Colladay and V.A. Kostelecký, Phys. Rev. D 55, 6760 (1997); 58, 116002 (1998).

[6] V.A. Kostelecký and S. Samuel, Phys. Rev. D 39, 683 (1989);
V.A. Kostelecký and R. Potting, Nucl. Phys. B359, 545 (1991).

[7] V.A. Kostelecký and R. Lehnert, Phys. Rev. D 63, 065008 (2001).

[8] J. Wess and B. Zumino, Nucl. Phys. B70, 39 (1974).

[9] A. Salam and J. Strathdee, Nucl. Phys. B76, 477 (1974).

[10] R. Haag, J.T. Lopuszanski, and M. Sohnius, Nucl. Phys. B88, 257 (1975).

[11] J. Wess and B. Zumino, Phys. Lett. 49B, 52 (1974); L. O’Raifeartaigh, Nucl. Phys. B96, 331 (1975); E. Witten, ibid. B185, 513 (1981).

[12] J.S. Bell, Ph.D. thesis, Birmingham University, 1954; G. Lüders, K. Dan. Vidensk. Selsk. Mat. Fys. Medd. 28, 5 (1954); W. Pauli, in Niels Bohr and the Development of Physics, edited by W. Pauli (McGraw-Hill, New York, 1955). 